einstein

Official Publication of the Instituto Israelita de Ensino e Pesquisa Albert Einstein

ISSN: 1679-4508 | e-ISSN: 2317-6385
LEARNING BY IMAGES

\section{Novel coronavirus pneumonia and} acute pulmonary thromboembolism: casualty or causality?

Pneumonia pelo novo coronavírus e tromboembolismo pulmonar agudo: casualidade ou causalidade?

Gabriel Laverdi Beraldo', Eduardo Kaiser Ururahy Nunes Fonseca', Patrícia Yokoo', Marina Justi Rosa de Matos'1, Marcela Emer Egypto Rosa', Murilo Marques Almeida Silva', Rodrigo Caruso Chate', Hamilton Shoji', Walther Yoshiharu Ishikawa'

${ }^{1}$ Hospital Israelita Albert Einstein, São Paulo, SP, Brazil.

DOI: 10.31744/einstein_journal/2020AI5750
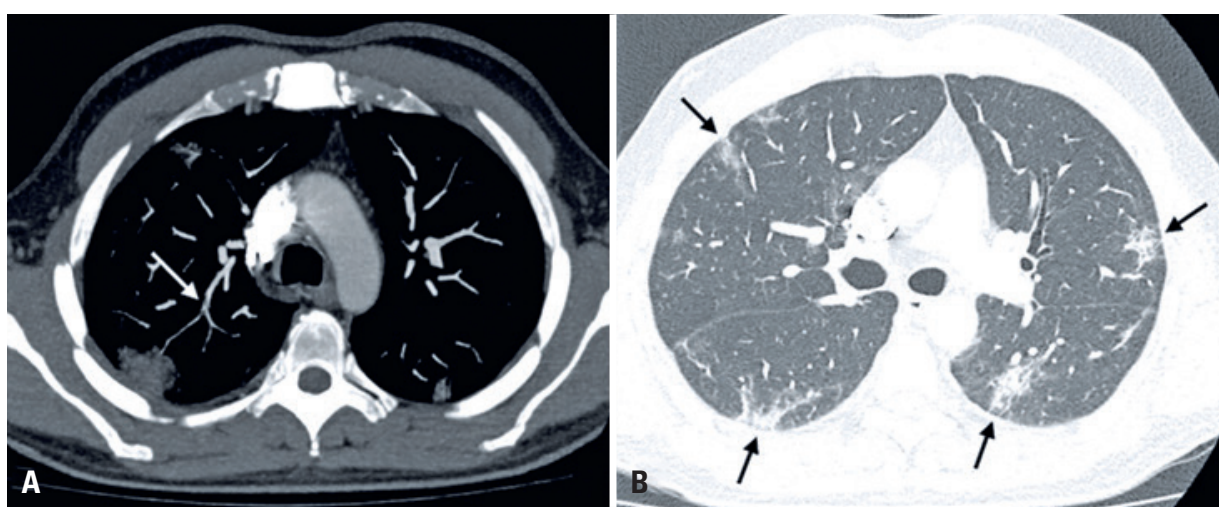

Figure 1. Computed tomography pulmonary angiography (49-year-old patient). (A) Axial mediastinal window shows filling defects of segmental and subsegmental artery branches of the right superior lobe (white arrows), findings compatible with pulmonary embolism. (B) Axial pulmonary window reveals multiple ground-glass opacities and thickened interlobular septae with bilateral and peripheral distribution (black arrows), findings consistent with coronavirus pneumonia
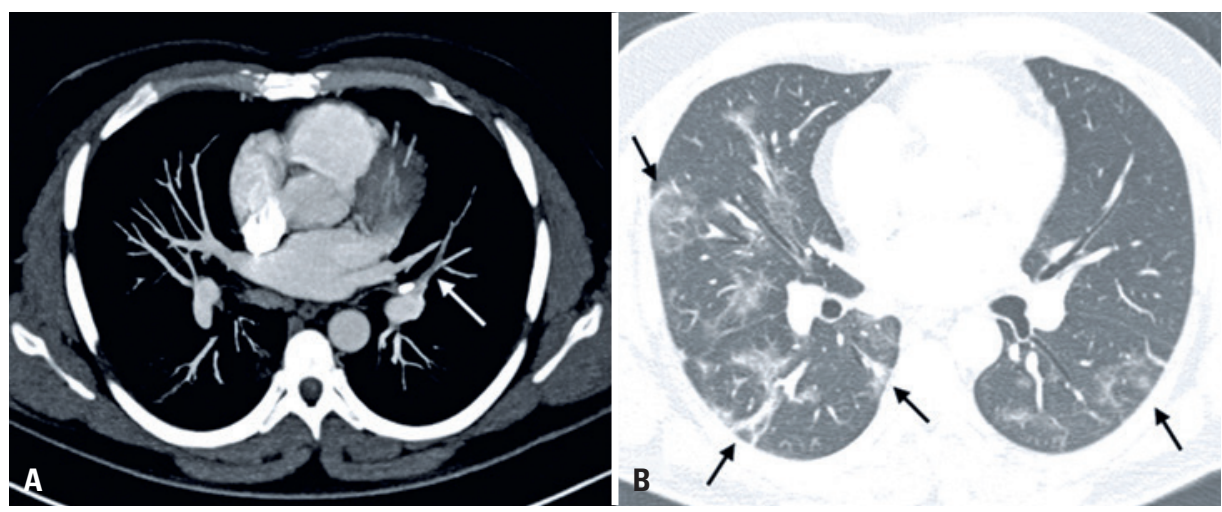

Figure 2. Computed tomography pulmonary angiography (41-year-old patient). (A) Axial mediastinal window shows lingular segmental pulmonary embolism (white arrows). (B) Axial pulmonary window reveals multiple ground-glass opacities, thickened interlobular septae, and parenchymal bands with bilateral and mainly peripherally distribution, findings consistent with COVID-19 pneumonia 
A great concern in the current global scenario, the pandemic caused by the novel coronavirus (COVID-19) has been one of the main reasons for chest computed tomography requests, both in early evaluation or late control. COVID-19 patients present an increased D-dimer, a finding likely related to hypercoagulability status and marker of worse prognosis. ${ }^{(1)}$ We report two cases with laboratory confirmation of coronavirus and acute pulmonary embolism - an association already described. ${ }^{(2,3)}$

A 49-year-old man who underwent a computed tomography pulmonary angiography (CTPA) due to a worsening of dyspnea, newly onset of chest pain and D-dimer elevation (711ng/dL) on the seventh day of hospitalization. A filling defect was observed on the segmental artery branch in the right superior lobe, a compatible finding with pulmonary embolism (Figure $1 \mathrm{~A})$, as well as typical changes caused by coronavirus pneumonia (Figure 1B). ${ }^{(4,5)}$

A 41-year-old man with worsening of dyspnea and D-dimer elevation $(1.427 \mathrm{ng} / \mathrm{dL})$ on the sixth day of hospitalization. A CTPA was performed, confirming pulmonary embolism on the lingular segmental branch (Figure 2A), in addition to the typical findings of COVID-19 pneumonia (Figure 2B). (4,5) $^{-19}$

Similar to other inflammatory processes, the COVID-19 pneumonia causes endothelial dysfunction and increases pro-coagulation activity. ${ }^{(6)}$ The elevation of D-dimer due to the infection is a finding that marks poor prognosis (higher hospitalization and death rates). ${ }^{(1)}$ There are reports of other cases of pulmonary embolism among these patients, however, this association is still unclear. ${ }^{(2,3)}$ We must be alert to pulmonary embolism in patients infected by COVID-19, particularly in cases of sudden worsening of dyspnea, given that overlap of clinical and laboratory findings makes the correct diagnosis more difficult, impacting on the morbidity and mortality.

\section{AUTHORS' INFORMATION}

Beraldo GL: http://orcid.org/0000-0002-9191-737X

Fonseca EK: http://orcid.org/0000-0002-0233-0041

Yokoo P: http://orcid.org/0000-0002-3493-8641

Matos MJ: http://orcid.org/0000-0002-2133-0434

Rosa ME: http://orcid.org/0000-0003-0423-3402

Silva MM: http://orcid.org/0000-0003-3748-5649

Chate RC: http://orcid.org/0000-0002-4193-7647

Shoji H: http://orcid.org/0000-0002-3701-4647

Ishikawa WY: http://orcid.org/0000-0002-5909-5126

\section{REFERENCES}

1. Wang D, Hu B, Hu C, Zhu F, Liu X, Zhang J, et al. Clinical characteristics of 138 hospitalized patients with 2019 novel coronavirus-infected pneumonia in Wuhan, China. JAMA. 2020;323(11):1061-9.

2. Xie Y, Wang X, Yang P, Zhang S. COVID-19 Complicated by acute pulmonary embolusm. Radiology: Cardiothoracic Imaging. 2020;2(2):e200067.

3. Danzi GB, Loffi M, Galeazzi G, Gherbesi E. Acute pulmonary embolism and COVID-19 pneumonia: a random association? Eur Heart J. 2020;41 (19):1858.

4. Chung $M$, Bernhein $A$, Mei $X$, Zhang $N$, Huang $M$, Zeng $X$, et al. CT imaging features of 2019 novel coronavirus (2019-nCoV). Radiology. 2020;295(1):202-7.

5. Lei J, Li J, Li X, Oi X. CT imaging of the 2019 novel coronavirus (2019-nCoV) Pneumonia. Radiology. 2020;295(1):18.

6. Ishiguro T, Matsuo K, Fujii S, Takayanagi N. Acute thrombotic vascular events complicating influenza-associated pneumonia. Respir Med Case Rep. 2019;28:100884. 